\title{
Schotterpetrologie und deren relativ-stratigraphische Anwendbarkeit im Aaretal südlich von Bern (Schweiz)
}

\author{
Von Christian Schlüchter, St. Catharines
}

Mit 3 Abbildungen

\begin{abstract}
$\mathrm{Z}$ u s a m m e $\mathrm{n}$ a s s u ng. Schotterpetrologische Untersuchungen im Aaretal südlich von Bern zeigen, daß die petrologische Zusammensetzung der Fraktion $>2 \mathrm{~cm}$ von älteren zu jüngeren Schotterakkumulationen gesetzmäßig ändert. Die ältesten, bis jetzt nachgewiesenen pleistozänen Schotter im Aaretal, die Bümbergschotter, sind reich an aufgearbeitetem Mollassematerial $(>15 \%)$, welches in den letzteiszeitlichen Münsingenschottern, bzw. spätglazialen Wichtrachschottern $<10 \%$ beträgt (Abb. 2). Bei den Münsingenschottern (Obere und Untere als Einheit betrachtet) kann östlich und westlich des heutigen Aarelaufes eine verschiedene Fazies in der Geröllführung nachgewiesen werden (Abb. 3). Das von CADISCH (1928) beobachtete Widerspiegeln der Heraushebungs- und Erosionsgeschichte des Alpenkörpers in der Petrologie der Molassesedimente gilt auch für die petrologische Geröllzusammensetzung der mittel- bis jungpleistozänen Schotterakkumulationen.

$\mathrm{Su} \mathrm{m}$ m a ry. Pebble petrological investigations on gravel deposits in the Aar River Valley, south of Bern, Switzerland, show a significant change in the petrological composition of the fraction $>2 \mathrm{~cm}$ : the oldest gravels (Bümbergschotter), deposited during the glaciation second before last, have a higher content of reworked material from the Miocene molasse (minimum of $15 \%$ ), wherelse Upper Münsingenschotter or Wichtrachschotter resp., show a maximum content of reworked molasse of $10 \%$. The sediments of the Kies-Sand-Komplex von Kleinhöchstetten are of intermediate composition (Fig. 2). Considering Upper and Lower Münsingenschotter as a whole, a pebble petrological facies east of the present Aar River can be distinguished from a facies west of it (Fig. 3). CADISCH (1928) stated, that the history of the uplift and erosion of the Alps is reflected in the petrological composition of the molasse sediments. This observation is also true for the Middle- to Joungpleistocene conglomerates.
\end{abstract}

\section{Einleitung und Historisches}

Quartärgeologische Untersuchungen (Geröllpetrologie und -morphometrie, KorngröBenanalysen) während der Jahre 1969-73 haben eine komplexe Abfolge von Schotterkörpern aufgezeigt, welche in wohldefinierte lithostratigraphische Einheiten gegliedert werden können. Die einzelnen Schotterkörper haben, wie wir sehen werden, ein geröllpetrologisch charakteristisches Spektrum, das relativ-stratigraphisch ausgewertet werden kann.

Erste Angaben über die geröllpetrologische Zusammensetzung der Aaretalschotter stammen von GERBER (1915). BECK (1922 bis 1943) bearbeitete die Aaretalschotter im wesentlichen vergleichend-lithostratigraphisch. An die Untersuchungen von Gerber anknüpfend, benutzt RuTsCH $(1928,1958)$ die petrologische Zusammensetzung der Schotter wieder als Charakteristikum. Die Ausscheidung von geröllpetrologisch definierten Schottertypen gelang nur, dank der großräumigen Aufschlüsse, durch konsequente Geröllzählungen in verschiedenen lithostratigraphischen Niveaus. Das Untersuchungsgebiet ist in Abb. 1 dargestellt. Die wichtigsten berücksichtigten Profile sind numeriert eingetragen, wobei chronostratigraphische Schlüsselprofile besonders bezeichnet sind (ScHLÜchteR $1973 \mathrm{~b})$. 


\section{Methodisches}

Die Geröllzählungen wurden, soweit möglich, im Aufschluß vorgenommen. Von Proben, die in $1 \mathrm{~m}$ Abstand durchs Profil entnommen wurden, ist die Fraktion $>2 \mathrm{~cm}$ abgesiebt worden. Pro Probe wurden 100 Gerölle nach den folgenden petrographischen Klassen bestimmt: Quarzite, rote Kristallingerölle, helle und grüne Kristallingerölle, kristalline Schiefer (metam. Kristallingerölle), grobkörnige Sandsteine, feinkörnige Sandsteine, mikritische Kalke, spätige Kalke, Echinodermenbrekzien, Dolomite und Rauhwacken.

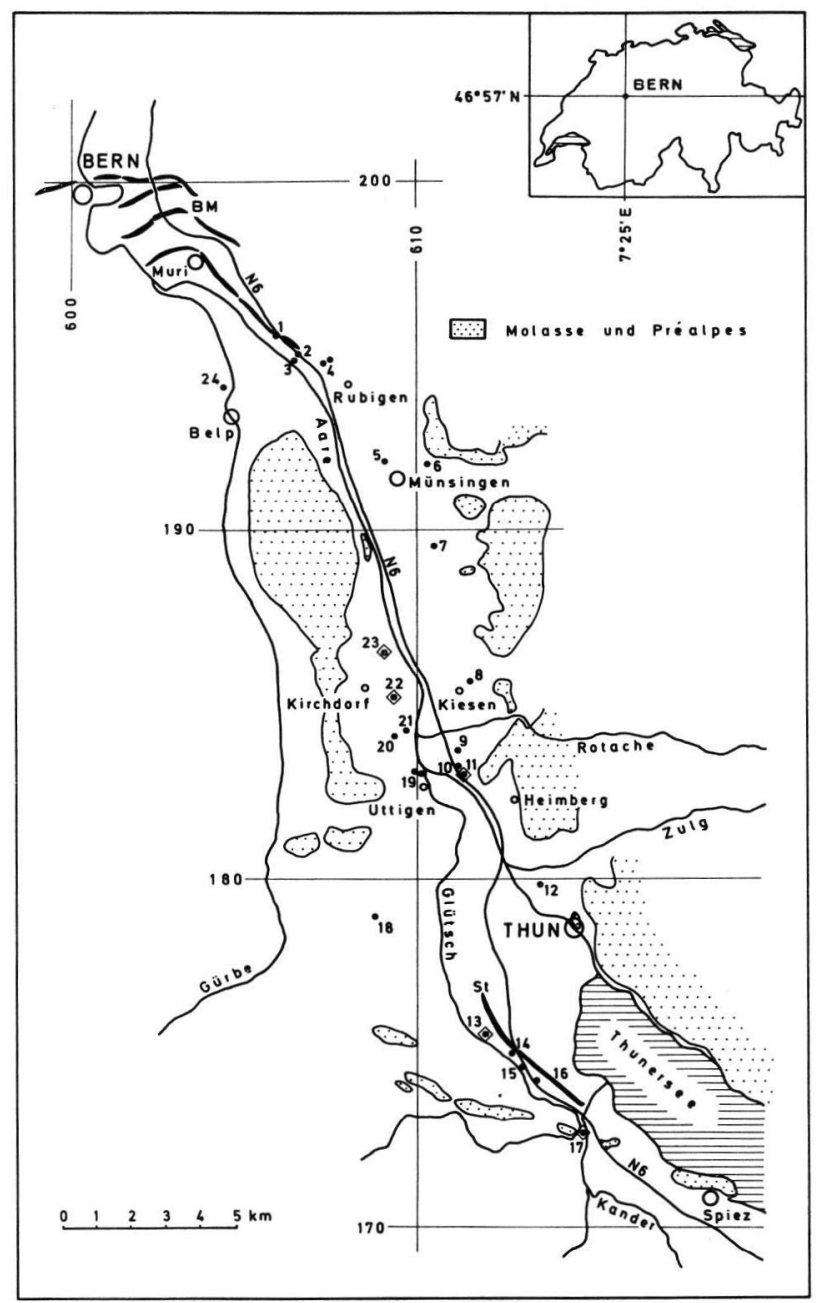

Abb. 1. Schlüsselkarte: Das Aaretal zwischen Bern und Spiez. - BM: Stauchendmoränen BernMuri, N 6: Nationalstraße 6 (Bern-Spiez), St: Seitenmoräne des Strättlig-Stadiums.

Profile: 1. Autobahnaufschluß Vorder Märchligen, 2. Stützmauer Raintalwald, 3. Raintalwald, 4. Kiesgruben N Rubigen, 5. Kiesgrube Anstalt Münsingen, 6. Mühle im Grabenthal, 7. Kiesgrube Niederwichtrach, 8. Kiesgrube Kiesen, 9. Kiesgrube Bümberg, 10. Autobahnaufschluß Grauestei, 11. Autobahnaufschluß Thungschneit/Räbeli, 12. Autobahnaufschluß Steffisburg, 13. Wässerifluh, 14. Scheibenstand Guntelsei, 15. Hurifluh, 16. Alte Schlyffi, 17. Kanderschlucht bei Hahni, 18. Schlupf, 19. Kiesgruben Uttigen, 20. Kiesgrube Buechlenacher, 21. Kiesgrube Ried, 22. Kiesgruben Jaberg, 23. Kiesgrube Thalgut, 24. Schulhausneubau Neumatt. (Profile von besonderer litho- und chronostratigraphischer Bedeutung sind mit einem Quadrat gekennzeichnet.) 
Zur Darstellung der Resultate in einem Dreiecksdiagramm ABC wurden folgende Klassengruppen gebildet (Abb. 2 und 3):

$\mathrm{A}=$ mikritische Kalke + spätige Kalke + Echinodermenbrekzie + Dolomite.

Bei diesen „Kalk"geröllen handelt es sich um Gesteine aus den helvetischen und praealpinen Decken nördlich der Zentralmassive.

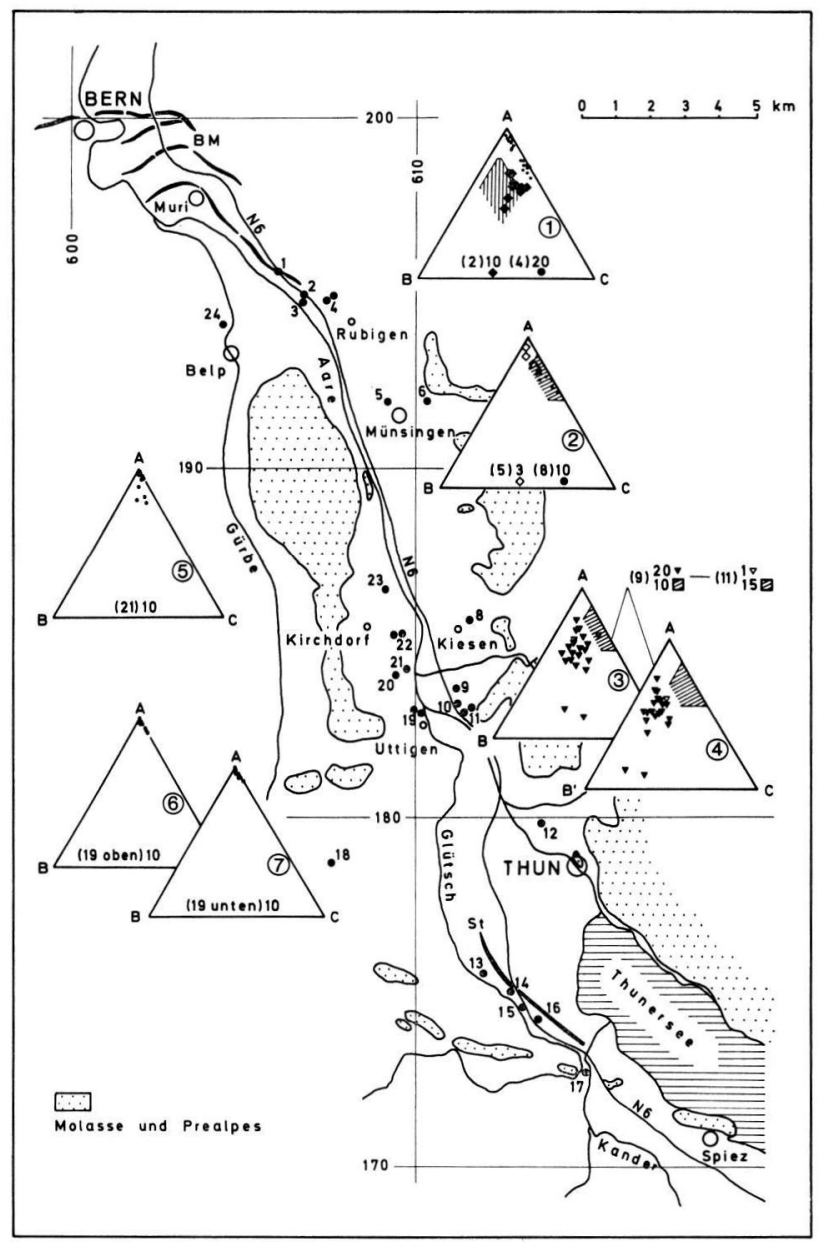

Abb. 2. Geröllpetrologie verschiedener Schotterkörper im Aaretal. Die Aufschlüsse sind analog Abb. 1 numeriert.

Im Dreieck bedeuten: $\mathrm{A}=$ Kalkgerölle, $\mathrm{B}=$ rote Kristallingerölle, $\mathrm{B}$ ' = rote Kristallingerölle + Quarzite (Dreieck 4), C = grüne Kristallingerölle. Eingeklammerte Zahl = Aufschlußnummer, zweite Zahl = Probenzahl (je 100 Gerölle).

Dreieck 1: Untere + Obere Münsingenschotter (Punkte), Kies-Sand-Komplex von Kleinhöchstetten (ausgefüllte Quadrate), Bümbergschotter (senkrecht schraffiertes Feld).

Dreieck 2: Obere Münsingenschotter: punktiert (Kiesgrube Kiesen), schraffiert (Thungschneitgebiet); Wichtrachschotter: Quadrate.

Dreiecke 3/4: Obere Münsingenschotter: schraffiertes Feld, Bümbergschotter: Dreiecke (leeres Dreieck $=$ Vergleichsprobe vom Räbeli).

Dreiecke 5-7: Obere Münsingenschotter im Gebiet Ried-Uttigen. 
$\mathrm{B}=$ rote Kristallingerölle.

Hier handelt es sich um aufgearbeitetes Material aus der miozänen, „bunten“ Molasse. Ebenfalls zu Gruppe B könnten die Quarzite gezählt werden, welche, mit wenigen Ausnahmen ( $\langle 5 \%$ des gesamten Quarzitgehaltes), aus der Molasse aufgearbeitet sind. Diese Werte $\mathrm{B}^{\prime}$ sind in Dreieck 4, Abb.2 berechnet worden.

$\mathrm{C}=$ helle und grüne Kristallingerölle.

Unter dieser Gruppe sind die hellen und grünen granitischen Gesteine zusammenge$\mathrm{faßt}$, welche heute in den kristallinen Massiven der Alpen erodiert werden; also wie die Gerölle unter A relativ „junge“ Komponenten der Lockergesteine sind. Bei dieser Gruppe muß auf eine Fehlerquelle hingewiesen werden, welche $10-15 \%$ der CWerte betragen kann: hier sind Gerölle mitgezählt worden, welche möglicherweise aus der bunten Nagelfluh aufgearbeitet, also Molassematerial sind. Bei weiteren schotterpetrologischen Untersuchungen wird unser Arbeitsschema dahin verfeinert werden müssen, daß Molasse- und Nichtmolasseanteile innerhalb der C-Werte getrennt werden. Das wird zu einer anderen Darstellung der Untersuchungsergebnisse führen, die mitgeteilt werden, sobald Untersuchungen aus einem weiteren Gebiet vorliegen.

\section{Petrologie der einzelnen Schotterkörper}

\subsection{Die $B$ ü mbergschot ter}

Im Profil Bümberg (Abb. 1 und 2, Profil 9) ist es möglich, die „Aelteren Aaretalschotter" Gerbers zu gliedern (SCHLÜCHTER 1973a). Tiefgründig verwitterte Schotter bilden hier und auch im Grauestei- und Thungschneit/Räbeli-Profil (Abb. 1 und 2, Profile 10 und 11) den Sockel der pleistozänen Lockergesteine. Es handelt sich um Deltaschotter mit den entsprechenden Übergußschichten. Diese Schotter sind im Thungschneit/Räbeli überdies glazigen aufgestaucht. - Chronostratigraphisch sind die Bümbergschotter einem ersten(?) glazialen Ereignis der vorletzten Eiszeit (Riß im klassischen Sinne) zuzuordnen (SCHLÜCHTER 1973b), sind somit zu den ältesten bis jetzt nachgewiesenen quartären Lokkergesteinen im Aaretal zu zählen.

Die geröllpetrologische Zusammensetzung ist in den Dreiecken 2 und 3, Abb. 2, dargestellt; damit wird gezeigt, wie das Verteilungsfeld der Bümbergschotterproben in beiden Darstellungen von den Analysenpunkten der Münsingenschotter abfällt. Die Bümbergschotter sind durch einen Mindestgehalt von $15 \%$ an Molassematerial charakterisiert, wobei die Gehalte von Kalkgeröllen und hellen und grünen Kristallingeröllen relativ stark schwanken.

Die beiden in den Dreiecken tief liegenden Bümbergschotterproben stammen aus deren verwittertem Dach, wo praktisch alle Kalk- und Dolomitgerölle verwittert und unbestimmbar sind. Die Bümbergschotter-Vergleichsprobe aus dem Thungschneit/Räbeli-Profil zeigt, wie der Quarzitgeröllanteil aus der miozänen Molasse lokal als eine wesentliche Bestimmungsgröße auftreten kann.

\subsection{Der Kies-Sand-Komplex von Kleinhöchstetten}

Im Gebiet von Raintalwald/Kleinhöchstetten (Abb. 1 und 2, Profil 2) folgt im Hangenden der Raintal Schlammoräne, an verschiedenen Stellen im Profil nachweisbar in deren sedimentgenetischer Kontinuität, eine Abfolge von Sand und Kies, die stark glazigen gestaucht ist. Es handelt sich bei diesem Kies-Sand-Komplex von Kleinhöchstetten um eine selbständige lithostratigraphische Einheit, die gegen Osten, im Gebiet von Rubigen, unter die Münsingenschotter abtaucht (SCHLÜCHTER 1973 b). Genetisch handelt es sich dabei um 
einen Rückzugsschotter, der später (während des ersten[?] und zweiten Vorstoßès der letzten Eiszeit) glazigen aufgestaucht worden ist. Chronostratigraphisch ist er in die ausgehende vorletzte Eiszeit zu stellen.

Im Zusammenhang mit der lithostratigraphischen Position des Kies-Sand-Komplexes von Kleinhöchstetten ist seine geröllpetrologische Zusammensetzung von Bedeutung (Abb. 2, Dreieck Nr. 1). Die Analysenpunkte der Kies-Sand-Komplex-Proben fallen z. T. in, aber auch außerhalb des Analysenfeldes der Bümbergschotter. Im Vergleich mit den Münsingenschotter-Proben aus dem gleichen Gebiet sind sie ebenfalls verschieden und tendieren zu einer Mittelwertstellung zwischen Bümbergschotter- und MünsingenschotterZusammensetzung.

\subsection{Die Münsingenschotter}

Die mächtigste Schotterablagerung im Aaretal zwischen Bern und Spiez bilden die Münsingenschotter (BECK, ohne Datum; Beck \& RuTsch 1958). Über ihre Verbreitung im Zusammenhang mit den damit verknüptten Grundmoränen gibt Abb. 3 einen Überblick. Die Münsingenschotter sind in eine Untere und eine Obere Einheit gliederbar, wobei die Untere einem ersten, die Obere einem zweiten letzteiszeitlichen glazialen Ereignis zugeordnet werden kann (Schlüchter 1973 a, 1973 c). Die Ablagerung der Unteren Münsingenschotter ist in die Zeit von 50-60000(?) B.P., jene der Oberen zwischen $19500-$ 18000 (?) B.P. zu stellen. Der Komplex der Unteren und der Oberen Münsingenschotter ist ein charakteristisches Beispiel eines Vorstoßschotters (BECK \& RuTSCH 1958, SchlüchTER 1973 a).

In unseren geröllpetrologischen Untersuchungen sind die Münsingenschotter vorläufig in Bezug auf ihre Zusammensetzung als Einheit betrachtet worden (Abb. 2), da es mit der angewendeten Methode noch nicht gelungen ist, eventuelle Unterschiede in deren Geröllzusammensetzung darzustellen. Als wesentliches Merkmal sei herausgehoben, daß sich die Münsingenschotter durch einen niedrigen Gehalt an Molassematerial (max. $10 \%$ ) gegenüber Bümbergschotter und Kies-Sand-Komplex von Kleinhöchstetten unterscheiden. Der Gehalt an hellen und grünen Kristallingeröllen kann in vereinzelten Proben, besonders im Thungschneit/Räbeli-Gebiet, bis gegen $45 \%$ betragen. Der hohe Gehalt an Kalkgeröllen und hellen und grünen Kristallingeröllen gibt den Münsingenschottern im Aufschluß eine charakteristische graublaue Farbe.

Auf eine interessante Beobachtung sei besonders hingewiesen: wie in Abb. 3 dargestellt, kann gezeigt werden, daß die geröllpetrologische Zusammensetzung der Münsingenschotter links und rechts der Aare verschieden ist. Links der Aare ist sie einheitlich: aufgearbeitetes Molassematerial und helle und grüne Kristallingerölle fehlen in vielen Proben. Interessant ist die Zusammensetzung von vier Geröllproben aus der Kiesgrube Ried (Profil 21, Abb. 1), welche unterhalb des Verteilungsfeldes der Münsingenschotter-Proben links der Aare fallen. Sie stammen aus einer annähernd Ost-West verlaufenden Rinnenfüllung, wobei hier offensichtlich Material aus dem Gebiet östlich des heutigen Aarelaufes herangeführt worden ist.

Die Ausbildung der beiden verschiedenen geröllpetrologischen Faziesbereiche der Münsingenschotter östlich und westlich des heutigen Aarelaufes kann folgendermaßen erklärt werden: Ostlich von Aare/Thunersee stehen mächtige Schichtpakete der miozänen bunten Molasse an (verantwortlich für höheren Gehalt an aufgearbeiteten Geröllen aus der Molasse) und die Zufuhr an Kristallingeröllen aus dem heutigen Kristallinbereich der Alpen scheint dort, in der zentralen Fließ- und Sedimentationsrichtung der Aare, größer gewesen zu sein. Währenddem westlich des heutigen Aarelaufes der Oberflächenanteil der miozänen Molassenagelfluh wesentlich zurücktritt, hier aber die Sedimentzufuhr aus dem Randgebiet der praealpinen Decken (eventuell auch aus Kander- und Simmetal(?) wichtig ist. 


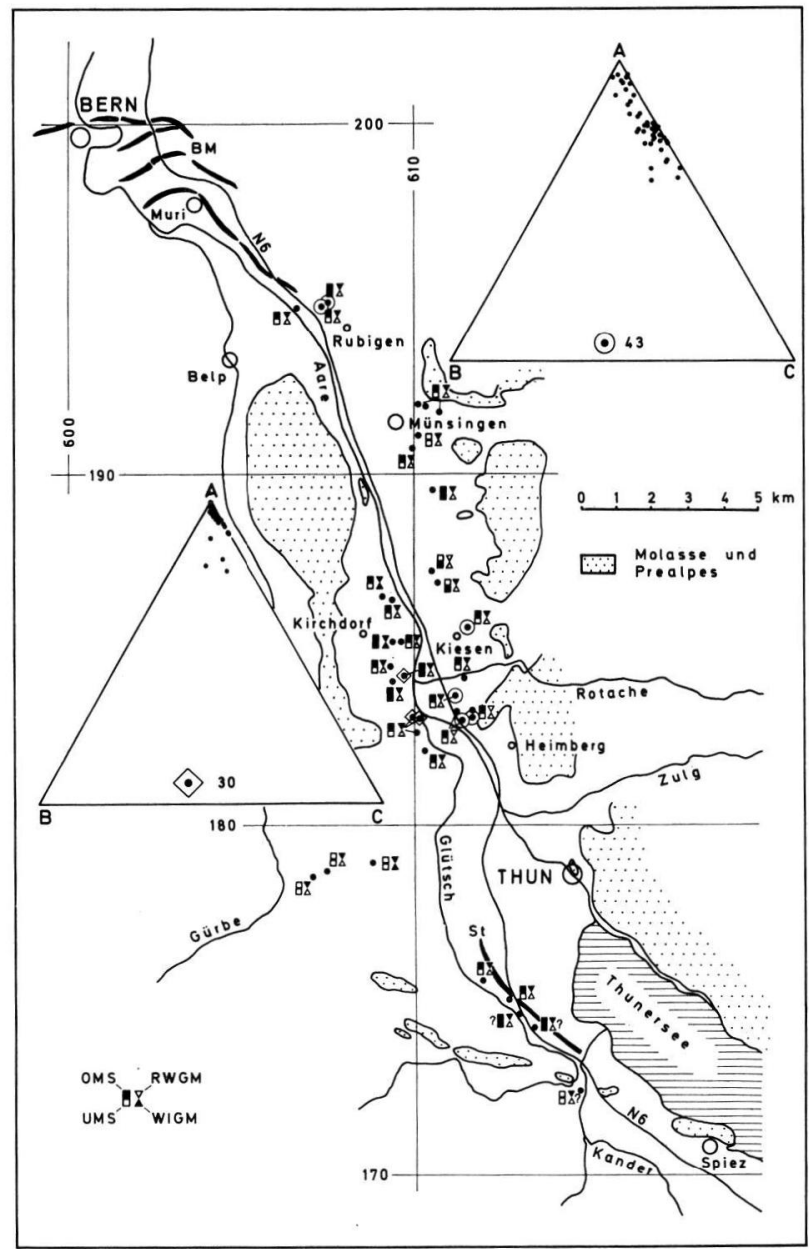

Abb. 3. Geröllpetrologische Zusammensetzung der Münsingenschotter links und rechts der Aare (Erklärung der Dreiecke: Abb. 2); und Verbreitung von Rotachewald Grundmoräne (RWGM), Obere Münsingenschotter (OMS), Würm I-Grundmoräne (WIGM) und Untere Münsingenschotter (UMS). Ausgefüllte Quadrat- und Dreiecksymbole: betreffende Ablagerung im bezeichneten Aufschluß nachgewiesen. Fragezeichen: lithostratigraphische Korrelation unsicher.

\subsection{Die Wichtrachschotter}

Der jüngste Schotterkörper des mittleren Aaretals, die Wichtrachschotter, bildet zwischen Rubigen und Wichtrach/Kiesen das komplexe System der Niederterrasse. Es handelt sich dabei um einen Rückzugsschotter im weitesten Sinne, dessen Genese an anderer Stelle ausführlich dargestellt worden ist (ScHLÜCHTER, 1973). In der Kiesgrube bei der Heilund Pflegeanstalt Münsingen (Abb. 1, Profil 5) sind zum Vergleich drei Geröllzählungen durchgeführt worden. Die Resultate sind in Dreieck 2 Abb. 2 eingetragen. Dabei wird gezeigt, daß die Zusammensetzung der Wichtrachschotter mit jener der Münsingenschotter praktisch identisch ist. Freilich fallen die Analysenpunkte etwas oberhalb des Verteilungsfeldes der Münsingenschotter des mittleren Aaretals (Dreiecke 2 und 3, Abb. 2), fallen aber 
zusammen mit den Analysen der Oberen Münsingenschotter aus der westlichen der beiden Kiesgruben bei Rubigen (Obere Münsingenschotter-Analysenpunktgruppe im Dreieck 1, Abb. 2).

\section{Stratigraphische Folgerungen}

Die Entwicklung der geröllpetrologischen Zusammensetzung der Schotterkörper im Aaretal südlich von Bern von den vorletzteiszeitlichen Bümbergschottern, über den KiesSand-Komplex von Kleinhöchstetten und die Unteren und Oberen Münsingenschotter zu den spätglazialen Wichtrachschottern zeigt: je jünger ein Schotterkörper ist, desto höher ist sein Gehalt an Kalkgeröllen der helvetischen und praealpinen Decken einerseits und an Geröllen jener kristallinen Einheiten der Alpen, die im mittleren und jüngeren Pleistozän zur Erosion kamen andererseits. Der Gehalt an aufgearbeitetem Molassematerial ist in den jungen Münsingen- und Wichtrachschottern ebenfalls niedriger als in den vorletzteiszeitlichen Bümbergschottern und den Sedimenten des Kies-Sand-Komplexes von Kleinhöchstetten.

Nach unseren heutigen Kenntnissen waren Oberstes Tertiär, Altpleistozän und die älteren Abschnitte des Mittelpleistozäns im Aaretal durch bedeutende Erosion gekennzeichnet. Seit dem Oberen Mittelpleistozän aber (ca. seit Beginn der vorletzten Eiszeit ?) wurden bedeutende Mächtigkeiten von fluvioglazialen, glazialen, fluviatilen und limnischen Lockergesteine abgelagert. Wie vorangehend gezeigt wurde, erfolgte die geröllpetrologische Entwicklung vom Liegenden zum Hangenden gesetzmäßig (Abb. 2), so daß diese zur relativen chronostratigraphischen Einstufung eines betreffenden Schotterkörpers verwendet werden kann. Lokale petrologische Schotterfazies, wie im Falle der Münsingenschotter (östlich/westlich des heutigen Aarelaufes) geben überdies genauere Hinweise über die Herkunft des betreffenden Sedimentmaterials. Nach den vorliegenden Untersuchungen dürfen die Ergebnisse von CADISCH (1928) dahin erweitert werden, daß sich die Heraushebungs- und Erosionsgeschichte des Alpenkörpers, die tektonische Geschichte des Hinterlandes, nicht nur in der Geröllfazies des Molassetroges, sondern bis hinauf in die jungpleistozänen Schotterakkumulationen widerspiegelt.

Es ist dem Autor cin Anliegen, Herrn Th. Kästli, Kies AG - Aaretal, verbindlichst für die Erlaubnis, in den Aaretalkiesgruben arbeiten zu dürfen und für das Interesse, das unseren Untersuchungen entgegengebracht wurde, zu danken. Ebenso verdankt sei das Verständnis und Entgegenkommen von Herrn F. Kilchenmann vom Autobahnamt des Kantons Bern. Besonders gedankt sei Herrn Prof. R. F. Rutsch, Bern, für seine stetige Förderung der Untersuchungen und Herrn Prof. G. Furrer, Zürich, für seine aufmunternde Kritik.

\section{Schriften}

Autobahnamt des Kantons Bern: Unpublizierte geotechnische Unterlagen des Autobahnbaues (Karten, Bohrungen, Analysen) - bis 1971.

BECK, P.: Der Alpenrand bei Thun. Gliederung der diluvialen Ablagerungen bei Thun. Bericht über die Exkursion ins Stockhorngebiet vom 29. bis 31. August 1922. - Eclogae Geologicae Helvetiae XVII/3, 1922.

— : Die Natur des Amtes Thun. - Das Amt Thun, Bd. 1, 1943.

— : Das Quartär. Das Pleistozän. - Manuskript, ohne Datum.

Beck, P. \& Rutsch, R. F.: Geologischer Atlas der Schweiz. Erläuterungen zu Atlasblatt 21. Kümmerly \& Frey, Bern 1958.

CaDisch, J.: Das Werden der Alpen im Spiegel der Vorlandsedimentation. - Geol. Rdsch. 19, 1928.

Gerber, E.: Uber ältere Aaretalschotter zwischen Spiez und Bern. - Mitt. naturforsch. Ges. Bern, Jg. $1914 / 15$.

Gasser, U. \& Nabholz, W. K.: Zur Sedimentologie der Sandfraktion im Pleistozän des schweizerischen Mittellandes. - Eclogae Geologicae Helvetiae, LXII/2, 1969. 
Rutsch, R. F.: Geologie des Belpberges. Beiträge zur Kenntnis der Stratigraphie, Paläontologie und Tektonik der Molasse südlich von Bern. - Mitt. naturforsch. Ges. Bern, Jg. 1928.

SCHLÜCHTER, C.: Die Münsingenschotter, ein letzteiszeitlicher Schotterkörper im Aaretal südlich Bern. - Festschr. Prof. Rutsch. Bull. Vereinig. Schweiz. Petroleumgeologen und -ingenieure, 39/96, 1973.

- : Geologische Untersuchungen im Quartär des Aaretals südlich von Bern (Stratigraphie, Paläontologie, Sedimentologie). Teil I (Text), Teil II (Figuren), Teil III (Fotos). 307 Seiten mit 42 Figuren, Karten und Tabellen und 112 Fotos. Manuskript. Dissertation, Philosophisch-Naturwissenschaftliche Fakultät der Universität Bern, Juli 1973.

- : Die Gliederung der letzteiszeitlichen Ablagerungen im Aaretal südlich von Bern (Schweiz). Z. für Gletscherkde. u. Glazialgeol., Band IX, (1-2), 123-141, 1973.

Manuskript eingeg. 20. 1. 1975.

Anschrift des Verf.: Dr. Christian Schlüchter, Department of Geological Sciences, Brock University, St. Catharines, Ontario, L 2 S 3 A 1, Canada. - Jetzige Adresse: Inst. für Grundbau der ETH, 8006 Zürich, Schweiz. 